\title{
Energy-Based Mechanical Model for Mixed Mode Failure of Laminated Composites
}

\author{
Seung J. Song* and Anthony M. Waas ${ }^{\dagger}$ \\ University of Michigan, Ann Arbor, Michigan 48109
}

\begin{abstract}
A nonlinear elastic foundation model has been developed to predict the mixed-mode (modes I and II) delamination failure of laminated composites. Two types of nonlinear elastic foundations were used to represent pure mode I and pure mode II components of the material failure ahead of the crack tip. One is a tension spring foundation for mode I component and the other is a shear spring foundation to represent the mode II component. Each spring foundation was characterized based on the linear elastic two-dimensional asymptotic solution of the strain field ahead of the crack tip and appropriate nonlinear constitutive laws. To predict the onset of crack propagation, the model employed an energy criterion as a failure condition derived from experimental trends of mixed-mode failure of laminated composites. Mode I, mode II, and mixed-mode fracture tests were performed to investigate the validity of the current model. A finite element was developed and incorporated into a computer code to simulate laboratory fracture tests, utilizing Timoshenko's first-order shear beam theory and a nonlinear constitutive law. The current model predicted the experimental results of load vs displacement curves closely. It also exhibited a satisfactory mode separation capability and was able to predict mode mixture results available in the literature.
\end{abstract}

\section{Introduction}

$\mathbf{L}$ AMINATED fibrous composites, in spite of their excellent mechanical and chemical properties, are known to be weak in planes that are perpendicular to the lamination plane. This weakness usually manifests itself in the form of delamination (or delayering) failure, a failure mode that has been and continues to be investigated thoroughly. Mode I, mode II, and mixed-mode fracture tests have been performed by many researchers to observe and understand delamination failure. A complete review of the literature is beyond the scope of this paper, however, the research work reported in Chai, ${ }^{1,2}$ O'Brien et al., ${ }^{3}$ Suo and Hutchinson, ${ }^{4}$ Hashemi et al., ${ }^{5,6}$ Poursartip and Chinatambi, ${ }^{7}$ and Kinloch et al., ${ }^{8}$ and the references contained therein serve as a summary of the work reported to date.

Linear elastic fracture mechanics (LEFM) based ideas and methodology, which are relatively well established for homogeneous and isotropic materials, have been naturally extended to analyze delamination growth problems in laminated composites. However, the mechanical complexity associated with tackling fracture problems of anisotropic layered materials has made this extension very complicated and, in some cases, almost impossible (for certain combination of materials characterized via the Dundurs mismatch parameters ${ }^{9}$ ).

Several models that adopt a spring foundation to mimic the material ahead of the crack which undergoes failure during crack propagation have been introduced in the past. Some of these representations are reported in Kanninen, ${ }^{10.11}$ Shilkrut, ${ }^{12}$ Ungsuwarungsri and Knauss, ${ }^{13}$ and Leonardi et al. ${ }^{14}$ In the present work, the simplicity associated with these models has been exploited and combined with salient features of the linear elastic twodimensional asymptotic crack tip strain field, to predict mixed-mode crack growth. The resulting spring foundation models have been validated with a systematic characterization of the spring properties using double cantilever beam (DCB) specimens. Two types of spring foundation models were previously developed depending on the failure criterion and characterization schemes. One is an energybased model $1^{15.16}$ and the other is a strength-based model. ${ }^{15,17}$ In the

Received Jan. 26, 1994; revision received June 20, 1994; accepted for publication June 24, 1994. Copyright (C) 1994 by Seung J. Song and Anthony M. Waas. Published by the American Institute of Aeronautics and Astronautics, Inc., with permission.

${ }^{*}$ Graduate Student, Department of Aerospace Engineering, Composite Structures Laboratory. Student Member AIAA.

${ }^{\ddagger}$ Associate Professor, Department of Aerospace Engineering, Composite Structures Laboratory. research reported here, the results from those investigations ${ }^{16}$ have been extended to mixed-mode delamination problems with an addition of a shear spring foundation. The advantage of an energy-based model is that the identification of the spring properties and the failure criterion adopted are both simple, thus making it easy to use in practice.

Experimental details of the laboratory mixed-mode fracture tests are given in Sec. II. The current mechanics model is formulated in Sec. III. A finite element is developed and explained in Sec. IV. Section V presents the results of the current model, and Sec. VI presents conclusions.

\section{Experiments}

Mode I, mode II and mixed-mode (I/II) fracture tests were performed using two different types of beam specimens (different composite systems) to investigate the delamination failure of laminated composites and to validate the current models.

\section{A. Specimen Preparation and Data Acquisition System}

The specimens were prepared by cutting E7T1/G40 and E719/ IM7 unidirectionally laminated 48-ply composite plates obtained from BP Chemical Co. The material properties for these composite systems are shown in Table 1. E7T1 is a thermoset and E719 is another type of thermoset having elastomer-like chemical structure. Each specimen had a 1-in.-long starter crack at the 8th, 16th, or 24th (center) ply, which was created with an insertion of a Teflon tape, prior to lay up and cure. A precrack was made by advancing the crack tip up to the crack length $a$. From this point the loading and unloading was applied repetitively using a Riehle displacement-controlled testing frame, and corresponding load vs displacement data were recorded. The resulting force and displacement at the loading point were measured using a 200 -lbf load cell and $\pm 5 \mathrm{~mm}$ linearly varying displacement transducer (LVDT), respectively. These data were recorded via an inhouse data acquisition system. The instantaneous crack-tip location was marked using a sharp knife while viewing the crack tip through a microscope on one side of the specimen which had been painted white to help find the crack tip more accurately.

\section{B. Three-Point Bending Tests}

Three-point bending tests were performed to obtain the bending stiffness of each type of beam using uncracked specimens. The equipment used in this test were a Riehle testing frame, a 200-lbf load cell, and a dial gauge. A shear factor was taken into account when the elastic modulus was calculated using Timoshenko beam theory. ${ }^{18}$ The elastic modulus in the transverse direction and the 
Table 1 Material properties of each beam specimen ${ }^{a}$

\begin{tabular}{lcccccc}
\hline Specimen & $V f$ & $\begin{array}{c}E b,{ }^{b} \\
\text { Gpa }\end{array}$ & $\begin{array}{lllll}E m, \\
\text { Gpa }\end{array}$ & $\begin{array}{l}E f, \\
\text { Gpa }\end{array}$ & Mode I & Mode II \\
\hline E7T1/G40 & $0.56 \sim 0.58$ & 116 & 4.1 & 286.8 & 326 & 905 \\
E719/IM7 & $0.56 \sim 0.58$ & 135 & 3.3 & 304.1 & 996 & 1440 \\
\hline
\end{tabular}

${ }^{a}$ From left: fiber volume fraction, avg. resin layer thickness, bending modulus, elastic modulus of resin and fiber, avg. fracture toughness.

${ }^{\mathrm{b}}$ Shear factor included. ${ }^{18}$

${ }^{c}$ Average values after taking out the hysteresis.

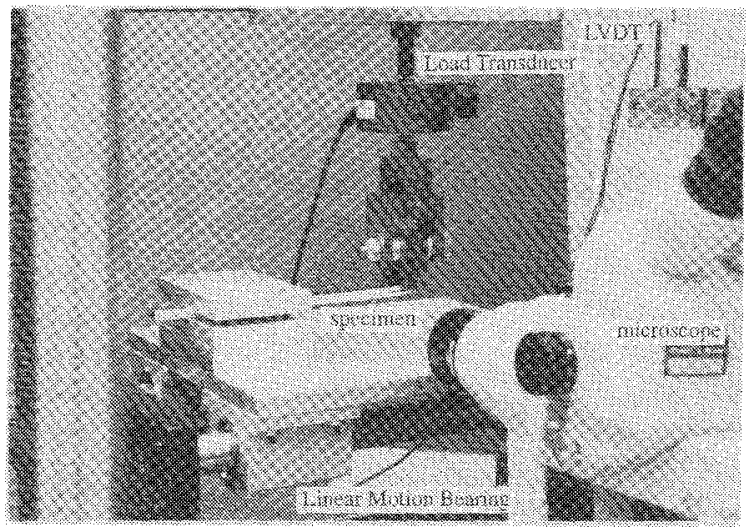

Fig. 1 Experimental setup of mode II and mixed-mode fracture tests showing beam specimen, clamping mechanism, microscope, LVDT, and load transducer.

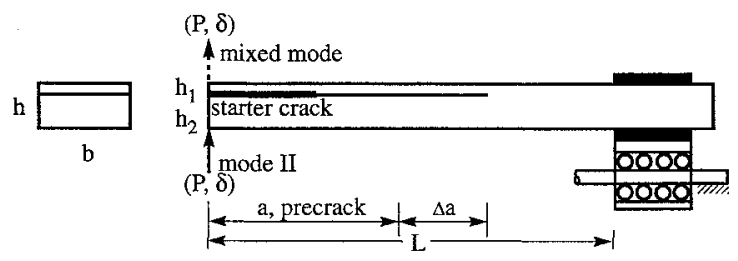

Fig. 2 Schematic of beam-type fracture specimen under mixed-mode or mode II failure.

through the thickness shear modulus were estimated using Halpin and Tsai's experimental formula. ${ }^{19}$

\section{Mode I, Mode II, and Mixed-Mode Fracture Tests}

Mode I fracture tests were performed previously and reported in Song and Waas. ${ }^{15-17}$ Figure 1 shows the experimental setup for mixed-mode and mode II fracture tests and Fig. 2 shows the corresponding schematic diagram of the specimen under delamination failure. The grips and clamping mechanism were specially designed so as to minimize unnecessary shear and moment arising from the loadings. The specimens were clamped at the end using two flat plates tightened by bolts, and the clamping plates were supported by four linear motion bearings. In this manner the original length of the specimen was retained the same for the purpose of simulation (the beam length should remain the same in the mathematical model) and the axial force arising from constraining the axial displacement due to the beam bending deformation was prevented. The loading was applied slowly at a speed of $0.3-0.5 \mathrm{~mm} / \mathrm{min}$ on a screw-driven Riehle testing frame, simulating quasistatic conditions.

Figures 3 and 4 show typical load vs displacement curves obtained from mode II and mixed-mode tests, respectively. As can be seen from these figures, the loading and unloading curves of mode II and mixed-mode tests have noticeable hysteresis. To investigate this phenomenon further, another test was performed in which a specimen without the artificial crack was loaded and unloaded several times up to the same maximum force under the same clamping mechanism used in the mode II and mixed-mode tests. The result is shown in Fig. 5. The specimen dissipated a noticeable amount of energy during the first loading, making the unloading path not follow the original loading path. The second loading curve did not follow

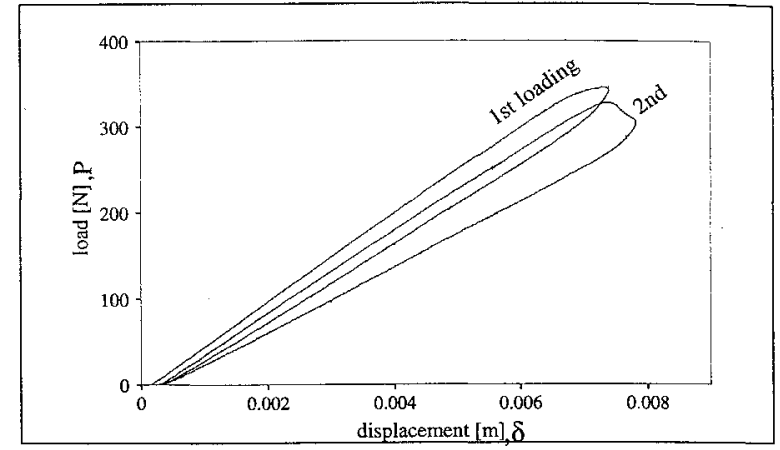

Fig. 3 Typical load vs displacement curves from mode II fracture tests.

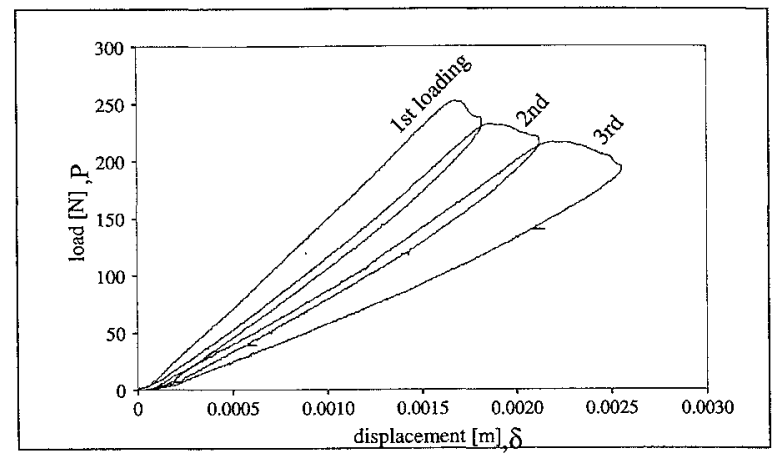

Fig. 4 Typical load vs displacement curves from mixed-mode fracture tests.

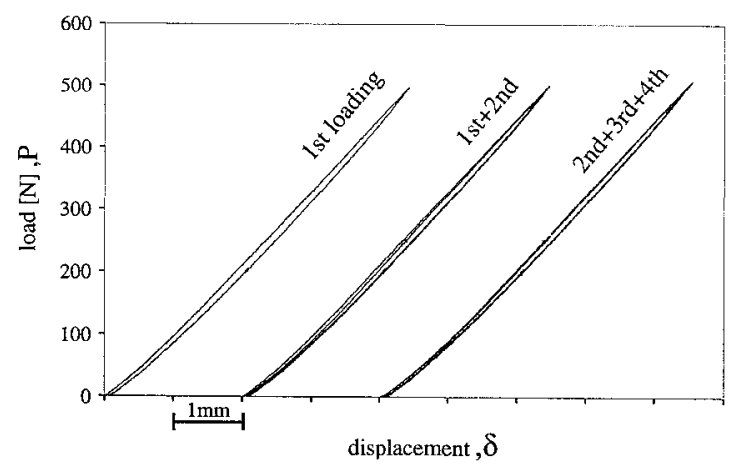

Fig. 5 Load vs displacement curves of uncracked cantilever beam specimen.

the previous unloading curve and further energy dissipation was noticed. The third and fourth loading and unloading curves followed the second loading and unloading curve. At the first loading, it is believed that the specimen underwent permanent microstructural and irreversible damage. However, at the second and consecutive loadings, the specimen, already permanently damaged as a part of the first loading, experiences repeatable microstructural change. This is perhaps why the second and consecutive loadings show the same amount of energy dissipation or hysteresis. To fully resolve this issue and ascribe a reason for this would require a thorough characterization of the composite microstructure, as well as an examination of internal friction and possibly slipping at the fiber/matrix interfaces. Although this aspect of the work is beyond the scope of the current investigation, it must be mentioned that similar findings have been observed and investigated by Lamborn and Schapery ${ }^{20}$ recently.

If the fracture toughness was to be calculated using the energy method $^{5}$ for this special test, it would produce a very high value (infinity) because the crack advance was zero. Therefore, it can be concluded that hysteresis from the loading and unloading curves in any kind of fracture tests should be excluded when calculating the fracture toughness. Figure 6 shows the fracture toughness data as a function of crack advance $(\triangle a)$ before and after, excluding the hysteresis. It shows that as the crack advance becomes smaller the 


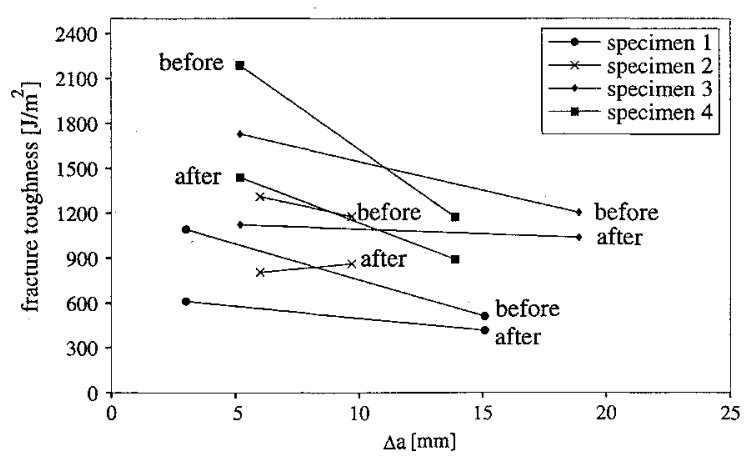

Fig. 6 Comparisons of toughness data between before and after taking out hysteresis from mode II and mixed-mode tests.

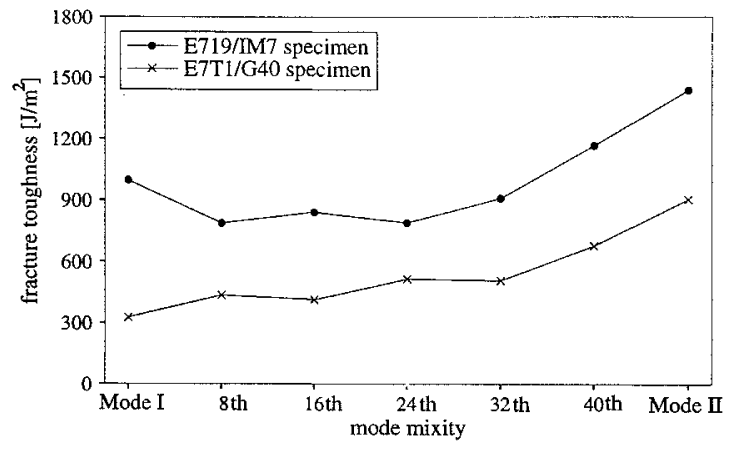

Fig. 7 Experimental results of fracture toughness as a function of mode mixture.

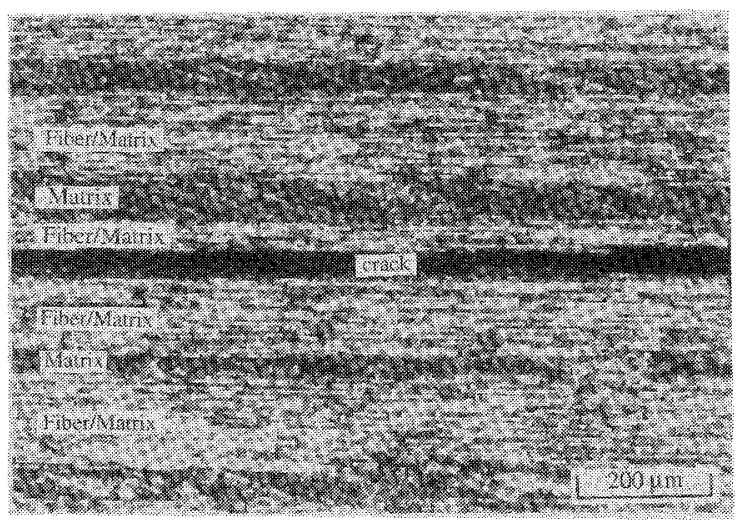

Fig. 8 Typical side view of cracked specimens showing intralaminar crack growth from mode II and mixed-mode tests.

toughness becomes large, thus it supports the argument stated previously. It also shows that the data, after taking out the hysteresis, become more independent of crack advance. Further, the amount of the hysteresis increased as the mode changed towards mode II in which the beam deformation was the largest. The resulting toughness data are shown in Fig. 7 as a function of mode mixity. The 8th, 16 th, etc. in the $x$ axis denote the crack location measured from the top of the specimen (Fig. 2).

After the tests, all of the cracked specimens were examined using a 50-1000 times magnification microscope. Figure 8 shows a typical side view of the crack path observed from mixed-mode and mode II tests. The crack shifted into intralaminar failure in every mixed and mode II fracture test, whereas mode I tests always revealed interlaminar fracture. Figure 9 shows the switching of the failure mode from interlaminar crack growth to intralaminar crack growth. The figure shows the place where a mixed-mode loading started from an interlaminar precrack. This phenomenon can be explained easily by considering the stress state ahead of the crack tip, ${ }^{21}$ where the principal plane, under normal and shear stresses, is situated at an angle of $\theta$ from the beam axis, which becomes $45 \mathrm{deg}$ for pure mode II loading conditions. The crack in a real fracture test, however,

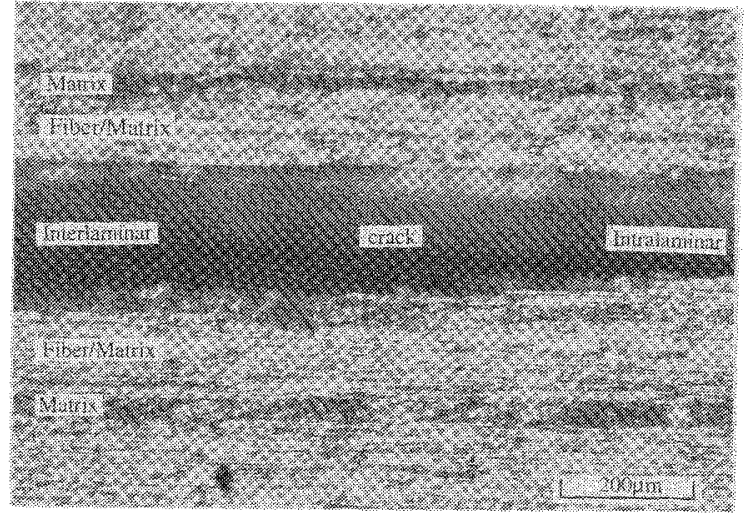

Fig. 9 Switching from interlaminar crack growth to intralaminar crack growth.

could not grow with such a large angle because there were much stiffer fibers running parallel to the beam axis, providing constraint for the crack to propagate within the resin.

\section{Formulation of the Model}

Figure 2 shows a schematic of a beam-type fracture specimen under mixed-mode or mode II failure. The specimen is a unidirectionally laminated composite beam having a precrack along the resin layer. At a certain value of external load, the crack starts to propagate, and the specimen experiences different mode mixity ahead of the crack tip depending on the geometry and loading conditions. Up to this point in the loading history, the specimen has undergone two different reactions against the external loading. One is a global or macro behavior over the entire volume of the specimen, and the other is a local or micro behavior near the crack tip. If these two types of different reactions can be separated out in the process of modeling, then the resulting boundary value problem becomes much easier to solve.

The macrobehavior of the specimen can be separated out and then solved readily using various mathematical models such as beam, plate, two-dimensional, or three-dimensional elasticity theories. The microbehavior can be separated out using a different mechanical model. In this study, a nonlinear elastic foundation was used to represent the microbehavior ahead of the crack tip. Since the specimen under consideration experiences mode mixity of mode I and mode II, two types of spring foundations will be used. One is a tension spring foundation to represent the mode I component, and the other is a shear spring foundation to represent the mode II component.

Figure 10 shows the spring foundation model which is an approximation of the physical fracture specimen shown in Fig. 2. The tension and shear spring foundations are made from an infinite number of Winkler type one-dimensional springs which have no interactions between any pair of springs. Each spring element has a nonuniform strain distribution along the length. The form of the strain distribution is assumed to be an approximation to the two-dimensional linear elastic asymptotic solution of the strain field near the crack tip. The tension and the shear spring, therefore, can assume the following strain distributions (Fig. 11):

$$
\begin{array}{lll}
\epsilon_{m}=\frac{A_{1}}{\sqrt{|z|},} & 0^{*} \leq z \leq t, & -t \leq z \leq-0^{*} \\
\epsilon_{p}=\frac{B_{1}}{\sqrt{|z|},} & t \leq z \leq h_{1}, & -h_{2} \leq z \leq-t \\
\gamma_{m}=\frac{A_{2}}{\sqrt{|z|}}, & 0^{*} \leq z \leq t, & -t \leq z \leq-0^{*} \\
\gamma_{p}=\frac{B_{2}}{\sqrt{|z|}}, & t \leq z \leq h_{1}, & -h_{2} \leq z \leq-t
\end{array}
$$

where $t$ is one-half of the resin layer thickness and $A_{1}-B_{2}$ are parameters to be determined. The subscripts $m$ and $p$ stand for the resin layer containing the crack tip and the rest of the beam part, treated as a homogenized structure, respectively. Here, $0^{*}$ denotes a 


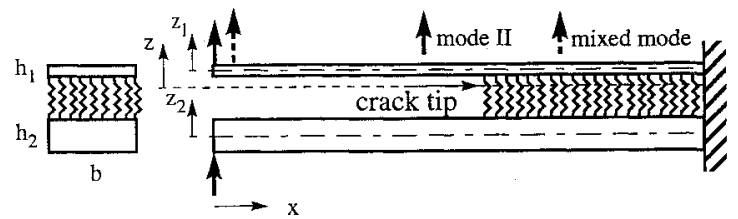

Fig. 10 Nonlinear elastic foundation model (tension and shear spring foundations).

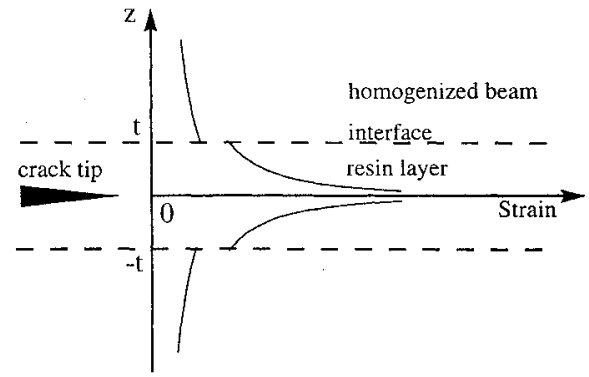

Fig. 11 Schematic of strain distribution throughout the spring length.

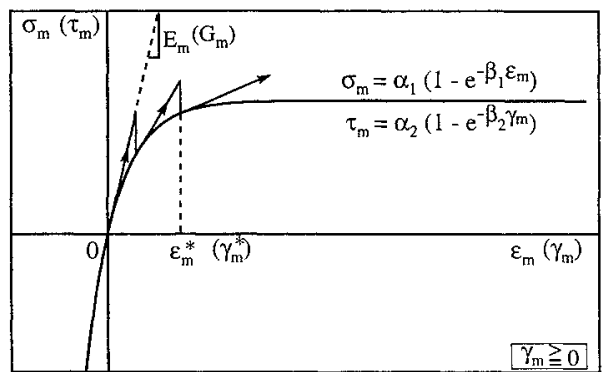

Fig. 12 Constitutive law of the resin layer and its schematic of multilinear model.

very small positive number close to 0 , and its meaning adopted in the context of the numerical solution is explained in Sec. IV.

The following form of the constitutive law is proposed (Fig. 12) so that the energy density of the spring at the crack tip becomes finite even though there is a stress singularity at $z=0$. In addition, by taking this form of constitutive law, the stress distribution along the $x$ axis ahead of the crack tip follows the form of a Dugdale model..$^{22}$

$$
\begin{array}{cr}
\sigma_{m}=\alpha_{1}\left(1-e^{-\beta_{1} \epsilon_{m}}\right), & 0^{*} \leq z \leq t, \quad-t \leq z \leq-0^{*} \\
\sigma_{p}=E_{p} \epsilon_{p}, & t \leq z) \\
\tau_{m}=\alpha_{2}\left(1-e^{-\beta_{2} \gamma_{m}}\right), & 0^{*} \leq z \leq t, \quad-h_{2} \leq z \leq-t \\
\tau_{p}=G_{p} \gamma_{p}, & t \leq z \leq h_{1}, \quad-t \leq z \leq-0^{*}
\end{array}
$$

where $E_{p}$ and $G_{p}$ are Young's modulus in the transverse direction and the shear modulus in the $x-z$ plane of the beam specimen, respectively. ${ }^{19}$ The beam parts above and below the resin layer were assumed to deform in the linear range. To simplify the algebra, the specimen is loaded such that the shear strain $\gamma_{m}$ becomes always positive. The parameters $\alpha_{1}-\beta_{2}$ can be obtained by using two conditions that the initial slopes of the constitutive laws be equal to Young's or shear modulus of the resin, and that the maximum stress be equal to the bulk material's ultimate tensile or shear stress. The application of these conditions yields

$$
\begin{gathered}
\alpha_{1}=\sigma_{\max } \\
\beta_{1}=\frac{E_{m}}{\sigma_{\max }} \\
\alpha_{2}=\tau_{\max } \\
\beta_{2}=G_{m} / \tau_{\max }
\end{gathered}
$$

The beam part was solved using Timoshenko's first-order shear beam theory. Thus, the following displacement fields are assumed for each beam part:

$$
\begin{aligned}
& u_{1}\left(x, z_{1}\right)=u_{01}(x)+z_{1} \psi_{1}(x) \\
& u_{2}\left(x, z_{2}\right)=u_{02}(x)+z_{2} \psi_{2}(x)
\end{aligned}
$$

The shear springs are attached to the bottom and top surfaces of the upper and lower beams, respectively, and the tension springs are attached to the centroidal lines of the two beams. The attachment of tension springs to the beam surfaces instead of the centroidal lines does not contribute a significant difference to the predicted displacement for a thin beam; instead, it makes the algebra more complicated.

Now the parameters $A_{1}-B_{2}$ can be obtained by applying appropriate conditions that the stresses at the interface between the resin layer and homogenized beam part should be the same, and that the displacement of the beam should be equal to the total deformation of the spring foundations. The conditions can be stated as follows with an additional assumption that the stresses at the interface are in the linear range:

$$
\begin{gathered}
E_{m} \epsilon_{m}(z= \pm t)=E_{p} \epsilon_{p}(z= \pm t) \\
G_{m} \gamma_{m}(z= \pm t)=G_{p} \gamma_{p}(z= \pm t) \\
w_{1}(x)-w_{2}(x)=2 \int_{0^{*}}^{t} \epsilon_{m} \mathrm{~d} z+\int_{t}^{h_{1}} \epsilon_{p} \mathrm{~d} z+\int_{t}^{h_{2}} \epsilon_{p} \mathrm{~d} z \\
u_{1}\left(x,-\frac{h_{1}}{2}\right)-u_{2}\left(x, \frac{h_{2}}{2}\right) \\
=2 \int_{0^{*}}^{t} \gamma_{m} \mathrm{~d} z+\int_{t}^{h_{1}} \gamma_{p} \mathrm{~d} z+\int_{t}^{h_{2}} \gamma_{p} \mathrm{~d} z
\end{gathered}
$$

After applying these conditions, the following results are obtained.

$$
\begin{gathered}
B_{1}=\left(E_{m} / E_{p}\right) A_{1} \\
B_{2}=\left(G_{m} / G_{p}\right) A_{2} \\
A_{1}=\left[w_{1}(x)-w_{2}(x)\right] / \Omega_{1} \\
A_{2}=\frac{\left[u_{01}(x)-u_{02}(x)\right]-\left[\left(h_{1} / 2\right) \psi_{1}(x)+\left(h_{2} / 2\right) \psi_{2}(x)\right]}{\Omega_{2}}
\end{gathered}
$$

where

$$
\begin{aligned}
& \Omega_{1}=2\left[2\left(\sqrt{t}-\sqrt{0^{*}}\right)+\left(E_{m} / E_{p}\right)\left(\sqrt{h_{1}}+\sqrt{h_{2}}-2 \sqrt{t}\right)\right] \\
& \Omega_{2}=2\left[2\left(\sqrt{t}-\sqrt{0^{*}}\right)+\left(G_{m} / G_{p}\right)\left(\sqrt{h_{1}}+\sqrt{h_{2}}-2 \sqrt{t}\right)\right]
\end{aligned}
$$

Therefore, Eqs. (1-4) become

$$
\begin{gathered}
\epsilon_{m}=\frac{1}{\Omega_{1}} \frac{w_{1}(x)-w_{2}(x)}{\sqrt{z}} \\
\epsilon_{p}=\frac{1}{\Omega_{1}}\left(\frac{E_{m}}{E_{p}}\right) \frac{w_{1}(x)-w_{2}(x)}{\sqrt{z}} \\
\gamma_{m}=\frac{1}{\Omega_{2}} \frac{\left[u_{01}(x)-u_{02}(x)\right]-\left[\left(h_{1} / 2\right) \psi_{1}(x)+\left(h_{2} / 2\right) \psi_{2}(x)\right]}{\sqrt{z}} \frac{1}{\sqrt{z}}\left(\frac{G_{m}}{G_{p}}\right) \frac{\left[u_{01}(x)-u_{02}(x)\right]-\left[\left(h_{1} / 2\right) \psi_{1}(x)+\left(h_{2} / 2\right) \psi_{2}(x)\right]}{\sqrt{\Omega_{2}}}
\end{gathered}
$$

A failure criterion for the spring foundation is needed to predict the onset of crack propagation. Johnson and Mangalgiri ${ }^{21}$ performed a literature review on laboratory fracture tests of mixed-mode failure 
of laminated composites. They found general data trends and concluded that, for practical purposes, the following empirical formula for a failure criterion can be used:

$$
\left(\frac{G_{\mathrm{I}}}{G_{\mathrm{Ic}}}\right)^{m}+\left(\frac{G_{\mathrm{II}}}{G_{\mathrm{II}}}\right)^{n}=1, \quad m=n=1
$$

Fracture toughnesses obtained from pure mode I tests (DCB) and pure mode II tests (End Loaded Split) will be used as $G_{I c}$ and $G_{I c}$, respectively. $G_{\mathrm{I}}$ and $G_{\mathrm{II}}$ will be replaced by the energy per unit area absorbed by the tension and the shear spring foundations, respectively, and can be expressed as

$$
\begin{aligned}
G_{\mathrm{I}} & =2 \int_{0^{*}}^{t} \int_{0}^{\epsilon_{m}} \sigma_{m} \mathrm{~d} \epsilon_{m} \mathrm{~d} z+\int_{t}^{h_{1}} \int_{0}^{\epsilon_{p}} \sigma_{p} \mathrm{~d} \epsilon_{p} \mathrm{~d} z \\
& +\int_{t}^{h_{2}} \int_{0}^{\epsilon_{p}} \sigma_{p} \mathrm{~d} \epsilon_{p} \mathrm{~d} z \\
G_{\mathrm{II}} & =2 \int_{0^{*}}^{t} \int_{0}^{\gamma_{m}} \tau_{m} \mathrm{~d} \gamma_{m} \mathrm{~d} z+\int_{t}^{h_{1}} \int_{0}^{\gamma_{p}} \tau_{p} \mathrm{~d} \gamma_{p} \mathrm{~d} z \\
& +\int_{t}^{h_{2}} \int_{0}^{\gamma_{p}} \tau_{p} \mathrm{~d} \gamma_{p} \mathrm{~d} z
\end{aligned}
$$

Equation (29) will be used as a failure criterion of the spring foundations along with Eqs. (30) and (31). This failure criterion implies that, even though the tension or shear spring at the crack tip does not individually break when Eq. (29) is satisfied, the material at the crack tip is at the critical state corresponding to crack growth.

\section{Finite Element Implementation}

The finite element method was employed to obtain solutions of the current mechanical model. To incorporate the material nonlinearity into the finite element, the nonlinear constitutive law was linearized first. The material having a one-dimensional nonlinear constitutive relation, Eqs. (5) and (7), can be approximated using a multilinear constitutive law (Fig. 12) as

$$
\begin{gathered}
\sigma_{m}=E_{1} \epsilon_{m}+E_{2} \\
\tau_{m}=G_{1} \gamma_{m}+G_{2}
\end{gathered}
$$

where $E_{1}-G_{2}$ can be expressed as

$$
\begin{gathered}
E_{1}=E_{m} e^{-\beta_{1} \epsilon_{m}^{*}} \\
E_{2}=\alpha_{1}\left[1-\left(1+\beta_{1} \epsilon_{m}^{*}\right) e^{-\beta_{1} \epsilon_{m}^{*}}\right] \\
G_{1}=G_{m} e^{-\beta_{1} \gamma_{m}^{*}} \\
G_{2}=\alpha_{2}\left[1-\left(1+\beta_{2} \gamma_{m}^{*}\right) e^{-\beta_{2} \gamma_{m}^{*}}\right]
\end{gathered}
$$

The asterisk in this equation denotes a quantity related to the previous incremental step.

Figure 13 shows a three-noded finite element incorporating Timoshenko beam theory with a correction factor of 5/6 (Ref. 18). The potential energy of the linearized system of the element can be written as

$$
\Pi=U_{\text {beam }}+U_{\text {spring }}-W_{e}
$$

where $U_{\text {beam }}$ and $U_{\text {spring }}$ denote the strain energy of the upper and lower beams and the spring foundations, respectively, and $W_{e}$ is the

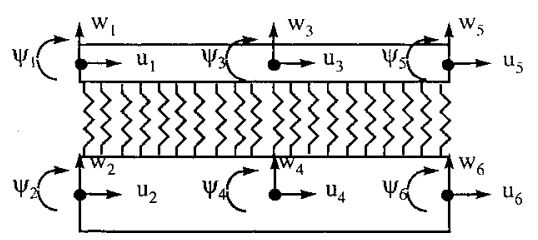

Fig. 13 Three-noded finite element with spring foundation. external work potential produced by nodal loads. Each of the terms is as follows:

$$
\begin{aligned}
& U_{\text {beam }}=\frac{1}{2} \int_{0}^{L}\left[E_{b} I_{1} \psi_{1, x}^{2}+\frac{5}{6} G_{p} A_{1}\left(w_{1, x}+\psi_{1}\right)^{2}\right] \mathrm{d} x \\
& +\frac{1}{2} E_{b} A_{1} \int_{0}^{L}\left(u_{01, x}\right)^{2} \mathrm{~d} x \\
& +\frac{1}{2} \int_{0}^{L}\left[E_{b} I_{2} \psi_{2, x}^{2}+\frac{5}{6} G_{p} A_{2}\left(w_{2, x}+\psi_{2}\right)^{2}\right] \mathrm{d} x \\
& +\frac{1}{2} E_{b} A_{2} \int_{0}^{L}\left(u_{02, x}\right)^{2} \mathrm{~d} x \\
& U_{\text {spring }}=2 b \int_{0}^{L} \int_{0^{*}}^{t} \int_{\epsilon_{m}^{*}}^{\epsilon_{m}} \sigma_{m} \mathrm{~d} \epsilon_{m} \mathrm{~d} z \mathrm{~d} x \\
& +b \int_{0}^{L} \int_{t}^{h_{1}} \int_{0}^{\epsilon_{p}} \sigma_{p} \mathrm{~d} \epsilon_{p} \mathrm{~d} z \mathrm{~d} x \\
& +b \int_{0}^{L} \int_{t}^{h_{2}} \int_{0}^{\epsilon_{p}} \sigma_{p} \mathrm{~d} \epsilon_{p} \mathrm{~d} z \mathrm{~d} x \\
& +b \int_{0}^{L} \int_{t}^{t} \int_{0}^{\gamma_{m}} \int_{\gamma_{m}^{*}}^{\gamma_{2}} \mathrm{~d} \gamma_{m} \mathrm{~d} z \mathrm{~d} x \\
& +b \int_{0}^{L} \int_{t}^{h_{1}} \int_{0}^{\gamma_{p}} \tau_{p} \mathrm{~d} \gamma_{p} \mathrm{~d} z \mathrm{~d} x+U_{p} \mathrm{~d} z \mathrm{~d} x \\
& +2 b \\
& +W_{e}=\sum_{i}^{\gamma_{p}} u_{i} P_{i}+w_{i} F_{i}+\psi_{i} M_{i} \\
& +b
\end{aligned}
$$

where $U^{*}$ is the strain energy, the resin layer containing the crack tip has absorbed up to $\epsilon^{*}$ and $\gamma^{*}$ in an incremental way. Instead of exact 0 in this study $1 \mu \mathrm{m}$ was used for $0^{*}$. The reason is related to the numerical implementation of the algorithm described. If the constitutive law is used in its original form (without a multilinear approximation), then the strain energy at the crack tip is bounded; however, this leads to a fully nonlinear finite element calculation which necessitates some form of linearization. Alternatively, the nonlinear constitutive law was replaced by a multilinear (piecewise linear) approximation as shown in Fig. 12. This approximation leads to an artificial singularity in the strain energy if exact 0 is used during the integration. Thus, this singularity was removed by using $0^{*}$, instead of 0 . The resulting finite element equations are linear (incrementally) and the solution procedure is then straightforward and efficient without sacrificing accuracy. The value of $0^{*}(1 \mu \mathrm{m})$ was chosen after careful examination ensuring that the multilinear model does not lead to unbounded results. In addition, as long as $0^{*}$ was significantly smaller (several orders of magnitude) than the characteristic lengths involved in the numerical calculation, the results were found to be insensitive to small deviations of $0^{*}$. Substitution of Eqs. (5-8) and (25-28) into Eq. (40) yields the following displacement related equation:

$$
\begin{aligned}
& U_{\text {spring }}=\Gamma_{1} \int_{0}^{L}\left[w_{1}(x)-w_{2}(x)\right]^{2} \mathrm{~d} x \\
& +\Gamma_{2} \int_{0}^{L}\left[w_{1}(x)-w_{2}(x)\right] \mathrm{d} x+\Gamma_{3} \int_{0}^{L}\left\{\left[u_{01}(x)-u_{02}(x)\right]\right. \\
& \left.-\left[\frac{h_{1}}{2} \psi_{1}(x)+\frac{h_{2}}{2} \psi_{2}(x)\right]\right\}^{2} \mathrm{~d} x+\Gamma_{4} \int_{0}^{L}\left\{\left[u_{01}(x)-u_{02}(x)\right]\right. \\
& \left.-\left[\frac{h_{1}}{2} \psi_{1}(x)+\frac{h_{2}}{2} \psi_{2}(x)\right]\right\} \mathrm{d} x+U^{* *}
\end{aligned}
$$


where $U^{* *}$ is a strain energy related to the previous $(n-1)$ th incremental step, which vanishes on differentiation with respect to the current step displacement fields. $\Gamma_{1}-\Gamma_{4}$ in the Eq. (42) are expressed as follows:

$$
\begin{aligned}
& \Gamma_{1}=\frac{b}{\Omega_{1}^{2}} \int_{0^{*}}^{t} \frac{E_{1}}{z} \mathrm{~d} z \\
& +\frac{1}{2} b E_{p}\left(\frac{E_{m}}{E_{p}}\right)^{2}\left(\log h_{1}+\log h_{2}-2 \log t\right) \\
& \Gamma_{3}=\frac{b}{\Omega_{2}^{2}} \int_{0^{*}}^{t} \frac{G_{1}}{z} \mathrm{~d} z \quad \frac{2 b}{\Omega_{1}} \int_{0^{*}}^{t} \frac{E_{2}}{\sqrt{z}} \mathrm{~d} z \\
& +\frac{1}{2} b G_{p}\left(\frac{G_{m}}{G_{p}}\right)^{2}\left(\log h_{1}+\log h_{2}-2 \log t\right) \\
& \Gamma_{4}=\frac{2 b}{\Omega_{2}} \int_{0^{*}}^{t} \frac{G_{2}}{\sqrt{z}} \mathrm{~d} z
\end{aligned}
$$

Now the displacement fields $u_{0}, w$, and $\psi$ can be approximated using quadratic interpolation in conjunction with a three-noded element

$$
\begin{aligned}
& u_{01}(x)=u_{1} N_{1}(x)+u_{3} N_{2}(x)+u_{5} N_{3}(x) \\
& u_{02}(x)=u_{2} N_{1}(x)+u_{4} N_{2}(x)+u_{6} N_{3}(x) \\
& w_{1}(x)=w_{1} N_{1}(x)+w_{3} N_{2}(x)+w_{5} N_{3}(x) \\
& w_{2}(x)=w_{2} N_{1}(x)+w_{4} N_{2}(x)+w_{6} N_{3}(x) \\
& \psi_{1}(x)=\psi_{1} N_{1}(x)+\psi_{3} N_{2}(x)+\psi_{5} N_{3}(x) \\
& \psi_{2}(x)=\psi_{2} N_{1}(x)+\psi_{4} N_{2}(x)+\psi_{6} N_{3}(x)
\end{aligned}
$$

where $N_{1}-N_{3}$ are the beam shape functions. ${ }^{16}$

After substituting the approximated deflections $u_{0}(x), w(x)$, and $\psi(x)$ into Eq. (38), the stationary potential energy theorem $(\delta \Pi=0)$ was applied to obtain an element matrix equation by differentiating the potential energy equation with respect to nodal displacements, $u_{1}-\psi_{6}$. The resulting $18 \times 18$ element stiffness matrix and $18 \times 1$ equivalent nodal loads were numerically integrated after approximating variables associated with the previous incremental step to be constants by taking average values within an element to save computation time. ${ }^{15-17}$

Figure 14 shows a schematic of the mesh generation. The mesh was generated in such a manner that the element numbering is dense near the crack tip, constant up to the final crack propagation point, and becomes sparse gradually from the point. The resulting global equation was numbered diagonally to save computer memory and computation time and was solved using a banded matrix solver. The numerical loading was applied by incrementing the displacement at the loading point. The crack propagation was achieved in a node-by-node advance scheme. In other words, the loading stops when the first spring at the crack tip has reached the failure criterion described in Sec. IIII, and the program calculates how much the crack has propagated and removes the spring foundation up to the nearest node of the new crack tip. This node-by-node crack propagation scheme, different from that of finding the exact location of the new crack tip and remeshing according to the new crack length, as reported by Song and Waas, ${ }^{15-17}$ has the advantage of having faster numerical convergence. A disadvantage of this scheme is that

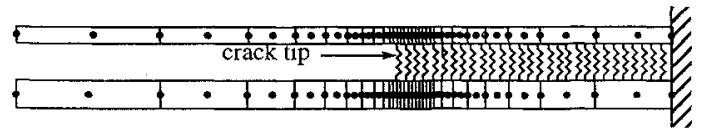

Fig. 14 Schematic of the mesh generation. a finer mesh distribution is required from the initial to final cracktip location. After reaching the final crack length in this manner where the loading switched to unloading in the experiment, the numerical loading also switched to unloading by decrementing the displacement. This whole process completes a loading and unloading curve. The smallest mesh size at the crack tip was about 0.1 $\mathrm{mm}$ and the increment of displacement was $5 \mu \mathrm{m}$. The computation time taken for one loop of the loading and unloading was approximately $1-3 \mathrm{~h}$ on a NeXT workstation computer. A much faster convergence (about 10 times) can be achieved if only critical loading and displacement at which the crack starts to propagate are sought, as the additional numerical scheme for crack propagation is not needed.

\section{Results}

A satisfactory numerical convergence was found for the total fracture toughness with an incremental size of $5 \mu \mathrm{m}$, total element number of 50, and smallest element size at the crack tip of $0.1 \mathrm{~mm}$.

The capability of the current model on mode separability has been tested for both types of specimens. The mode I and mode II components of the fracture toughness were calculated at the onset of the crack propagation during the simulation by calculating the strain energies absorbed by tension and shear spring foundations at the crack tip. The results for E7T1/G40 specimens are shown in Fig. 15, where 8th, 16th, etc. denote the crack location measured from the top of the specimen (Fig. 2), and those for E719/IM7 showed about the same results. The current model is seen to match the mode mixture very closely to the theoretical values calculated by Kinloch et al. ${ }^{8}$ For instance, the center crack mixed-mode specimen shows a mixture of $0.577-0.423$, or 1.36-1 from the current model, whereas Kinloch et al. ${ }^{8}$ predicts $4-3$, or $1.33-1$ based on the contribution of Hashemi et al. ${ }^{5}$

Figure 16 shows the prediction of the current model on the total fracture toughness of the mixed mode failure for two types of beam composites. As explained in Sec. III, the current model uses mode I and mode II fracture toughness data and follows a simple empirical formula obtained from the general data trends found by Johnson and Mangalgiri. ${ }^{21}$ The figure shows that the E7T1/G40 specimen followed the general trends closely, whereas the E719/IM7 specimen shows a little deviation from the trends. Therefore, the current model

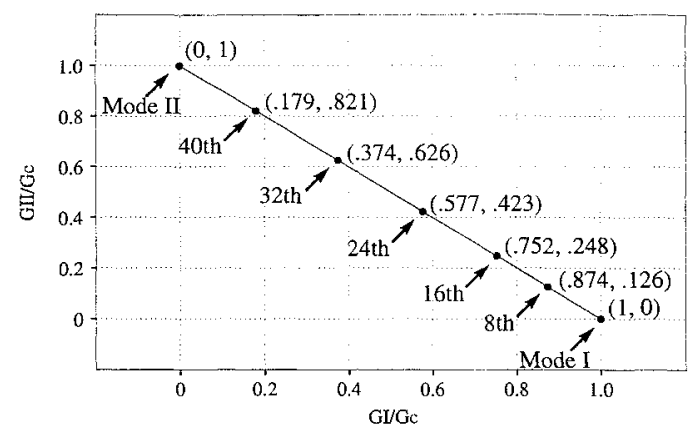

Fig. 15 Mode separation capability of the current model for E7T1/G40 specimen.

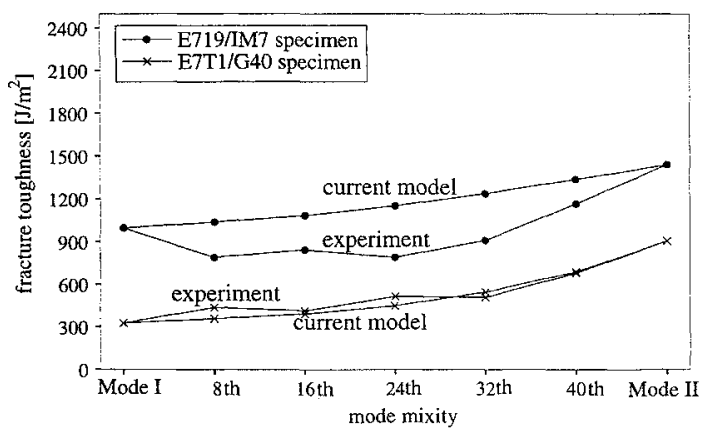

Fig. 16 Prediction of the mixed-mode toughness of the current model. 


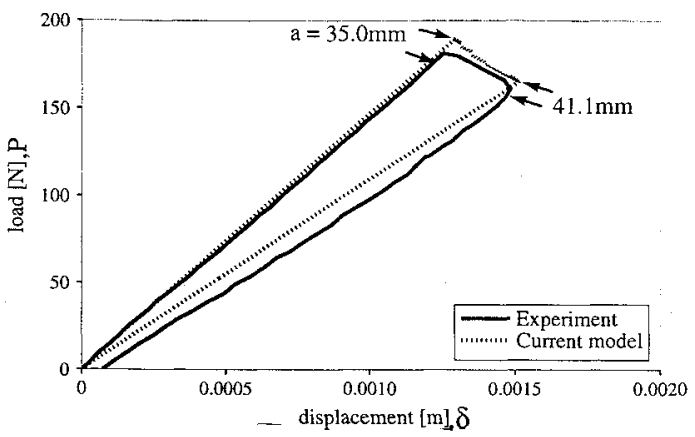

Fig. 17 Simulation results of the current model and experimental observations for E7T1/G40 specimen, 16th ply crack mixed mode.

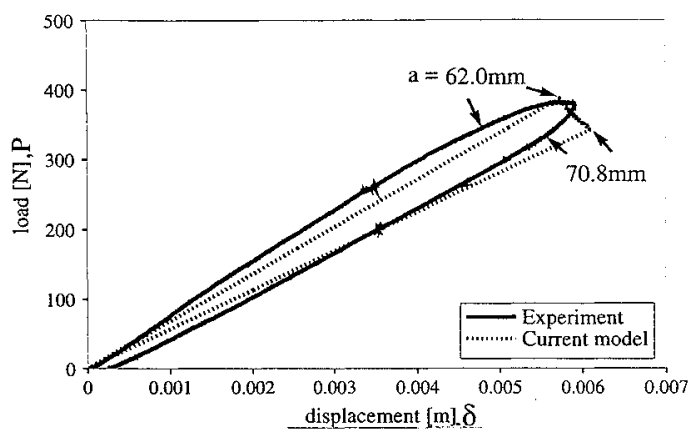

Fig. 18 Simulation results of the current model and experimental observations for E719/IM7 specimen, mode II.

will predict total mixed-mode fracture toughness accurately for the specimens which follow Eq. (29) closely. Figures 17 and 18 show the simulation results from the current model for the loading and unloading curves of mixed (16th-ply crack) and ELS (pure mode II) specimens. The current model also predicted the critical load and displacement of crack onset very accurately.

\section{Concluding Remarks}

The mechanical model that has been presented for mixed-mode fracture in layered composites, uses a tension and shear spring foundation to separate out microbehavior of the material ahead of the crack tip from the macrobehavior of the structure; tension springs are used to represent the mode I component and shear springs to represent the mode II component. The model uses the mode I and mode II fracture toughness data obtained from laboratory fracture tests for a given composite as an input and incorporates an empirical formula of general data trends as given by Johnson and Mangalgiri. ${ }^{21}$ An advantage of the presented model lies in its ability to separate out the mode mixture very easily, whereas the cantilever beam approach, ${ }^{23}$ for example, necessitates the use of many correction factors. Indeed, as was demonstrated, the separate contributions of mode I and mode II toughnesses are obtained as a result of the solution to the mechanics problem. In addition, the total toughness of mixed-mode failure as well as the critical loads and displacements at which the crack starts to propagate are also obtained as a part of the solution. The current model can be easily extended to practical crack problems, where the macrobehavior can be solved using well-established mechanics models such as beam, plate, shell, and two-dimensional elasticity theories, etc. and the microbehavior near the crack tip can be separated out and solved using the presented spring foundation model.

\section{Acknowledgments}

During the course of this work, the second author received partial funding from the Office of Naval Research (ONR), Mechanics
Division. We are thankful for this support. The interest and help of Y.D.S. Rajapakse from the ONR, Mechanics Division is gratefully acknowledged. The beam samples used in the study were donated by BP Chemicals Corp., California. We would like to thank E. Babayan and $\mathrm{A}$. Bosch for their support.

\section{References}

${ }^{1}$ Chai, H., "Shear Fracture," International Journal of Fracture, Vol. 37, 1988, pp. 137-159.

${ }^{2}$ Chai, H., "Interlaminar Shear Fracture of Laminated Composites," International Journal of Fracture, Vol. 43, 1990, pp. 117-131.

${ }^{3}$ O'Brien, T. K., Murri, G. B., and Salpekar, S. A., "Interlaminar Shear Fracture Toughness and Fatigue Thresholds for Composite Materials," Composite Materials: Fatigue and Fracture, Vol. 2, American Society for Testing and Materials, (ASTM) STP 1012, edited by P. A. Lagace, 1989, pp. $222-250$.

${ }^{4}$ Suo, Z., and Hutchinson, J. W., "Interface Crack Between Two Elastic Layers," International Journal of Fracture, Vol. 43, 1990, pp. 1-18.

${ }^{5}$ Hashemi, S., Kinloch, A. J., and Williams, J. G., "The Analysis of Interlaminar Fracture in Uniaxial Fibre-Polymer Composites," Proceedings of the Royal Society of London, Vol. A427, 1990, pp. 173-199.

${ }^{6}$ Hashemi, S., Kinloch, A. J., and Williams, G., "Mixed-Mode Fracture in Fiber-Polymer Composite Laminates," Composite Materials: Fatigue and Fracture, Vol. 3, American Society for Testing and Materials, ASTM STP edited by T. K. O'Brien, Philadelphia, PA, 1991, pp. 143-168.

${ }^{7}$ Poursartip, A., and Chinatambi, N., "Experimental Determination of the Mode I Behavior of a Delamination under Mixed-Mode Loading," Composite Materials: Fatigue and Fracture, Vol. 3, American Society for Testing and Materials, ASTM STP 1110, edited by T. K. O'Brien, 1991, pp. 187-209.

${ }^{8}$ Kinloch, A. J., Wang, Y., Williams, J. G., and Yayla, P., "The Mixed Mode Delaminations of Fiber Composite Materials," Composites Science and Technology, Vol. 47, 1993, pp. 225-237.

${ }^{9}$ Hutchinson, J. W., and Suo, Z., "Mixed Mode Cracking in Layered Materials," Advances in Applied Mechanics, Vol. 28, Academic Press, 1991

${ }^{10}$ Kanninen, M. F., "An Augmented Double Cantilever Beam Model for Studying Crack Propagation and Arrest," International Journal of Fracture, Vol. 9, No. 1, 1973.

${ }^{11}$ Kanninen, M. F., "A Dynamic Analysis of Unstable Crack Propagation and Arrest in the DCB Test Specimen," International Journal of Fracture, Vol. 10, No. 3, 1974

${ }^{12}$ Shilkrut, D., "Unilateral Contact Between a Geometrically Non-linear Plate and Elastic Foundation," Proceedings of the Second Meeting on Uni1ateral Problems in Structural Analysis, June 17-20, 1985.

${ }^{13}$ Ungsuwarungsri, T., and Knauss, W. G., "The Role of Damage-Softened Material Behavior in the Fracture of Composites and Adhesives," International Journal of Fracture, Vol. 35, 1987, pp. 221-241.

${ }^{14}$ Leonardi, A., Maceri, F., and Sacco, E., "Debonding of Bimodular Plates," International Series of Numerical Mathematics, Vol. 101, 1991, pp. 113-128.

${ }^{15}$ Song, S., and Waas, A., "A Nonlinear Elastic Foundation Model for Interlaminar Fracture of Laminated Composites," Composites Engineering, Vol. 3, No. 10, 1993, pp. 945-959.

${ }^{16}$ Song, S. J., and Waas, A. M., "A Spring Foundation Model for Mode I Failure of Laminated Composites based on an Energy Criterion," Journal of Engineering Materials and Technology (to be published).

${ }^{17}$ Song, S. J., and Waas, A. M., "Mode I Failure of Laminated Polymeric Composites," Engineering Fracture Mechanics (to be published).

${ }^{18}$ Whitney, J. M., Structural Analysis of Laminated Anisotropic Plates, Technomic, Westport, CT, 1987.

${ }^{19}$ Halpin, J. C., and Tsai, S. W., "Effects of Environmental Factors on Composite Materials," AFML-TR, 1969, pp. 67-423.

${ }^{20}$ Lamborn, M. J., and Schapery, R. A., "An Investigation of the Existence of a Work Potential for Fiber-Reinforced Plastic," Journal of Composite Materials, Vol. 27, No. 4, 1993.

${ }^{21}$ Johnson, W. S., and Mangalgiri, P. D., "Influence of the Resin on Interlaminar Mixed-Mode Fracture," Toughened Composites, American Society for Testing and Materials, ASTM STP 937, edited by N. J. Johnston, Philadelphia, PA, 1987, pp. 295-315.

${ }^{22}$ Dugdale, D. S., "Yielding of Steel Sheets Containing Slits," Journal of the Mechanics and Physics of Solids, Vol. 8, 1960, pp. 100-108.

${ }^{23}$ Williams, J. G., "On the Calculation of Energy Release Rate for Cracked Laminates," International Journal of Fracture, Vol. 36, 1988, pp. 101-119. 